\title{
A Novel Reconfigurable GaN Based Fully Solid-State Microwave Power Module for Communications/Radar Applications
}

\author{
Rainee N. Simons, Edwin G. Wintucky, and Seth W. Waldstein \\ NASA Glenn Research Center, 21000 Brookpark Road, Cleveland, OH 44135, USA
}

\begin{abstract}
The paper presents the design, integration, and performance of a novel S-/X-band, GaN based, fully solid-state microwave power module (SSMPM). The SSMPM includes diplexers, pre-amplifiers, a multistage medium power amplifier, SPDT switches, and CW/Pulsed high-power amplifiers. These components are synergistically integrated such that a single SSMPM is capable of being dynamically reconfigured to function as a S-/X-band amplifier for TT\&C/telecommunications for command/science date downlink and as a radar for remote sensing onboard a planetary exploration spacecraft. The SSMPM is capable of delivering $P_{\text {sat }}$ of $39 \mathrm{dBm}(8 \mathrm{~W} \mathrm{CW})$ at S-band, $P_{\text {sat }}$ of $46 \mathrm{dBm}(40$ $W \mathrm{CW})$ at X-band, and $P_{\text {sat }}$ of $>50 \mathrm{dBm}(>100 \mathrm{~W}$ Pulsed) at X-band. Index Terms-Gallium nitride, MMICs, satellite communications, SSPAs
\end{abstract}

\section{INTRODUCTION}

Historically, the term microwave power module (MPM) is associated with a small fully integrated self-contained RF amplifier that combines both solid-state and microwave vacuum electronics technologies (Fig. 1) [1]. In this paper, we present the research and development of a novel fully solidstate microwave power module (SSMPM), which is distinctly different from the above MPMs [2]. The SSMPM advances the state-of-the-art in spacecraft transmitters. Our effort leverages from the recent advances in RF wide bandgap semiconductor (WBGS) crystal growth, wafer preparation, device/circuit fabrication technologies and reliability studies that have resulted from the investment that DARPA has made with the U.S. industry [3]. As a result of DARPA investments, the past few years have witnessed significant progress in the development of GaN high electron mobility transistor (HEMT) on silicon carbide ( $\mathrm{SiC}$ ) substrate based monolithic microwave integrated circuits (MMICs) for RF power applications. The main advantages of GaN HEMT on SiC substrate is that it has power density as high as 12.2 watts/mm at X-band [4]. Thus, for a desired output power a GaN MMIC is smaller in size compared to a GaAs MMIC. In addition, $\mathrm{SiC}$ substrate has 8 to 10 times higher thermal conductivity than a GaAs substrate [5], which allows operating at higher junction temperature and also enhances thermal reliability by more efficiently conducting waste heat away from the junction.

A typical payload on an Earth and planetary exploration spacecraft includes S-band system for telemetry, tracking, and command (TT\&C) and a X-band or higher frequency system for science instruments and telecommunications. The role of the TT\&C system is receiving commands and downlinking spacecraft house-keeping data. Typical science instruments are scatterometers, radiometers, and radar for synthetic aperture imagers. The role of the telecommunication system is to downlink science data acquired by these instruments. The current state of practice uses two separate S-band and X-band amplifiers in

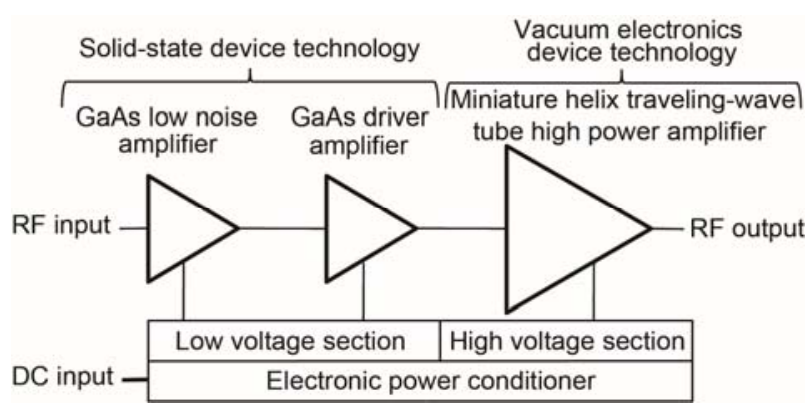

Fig. 1. Schematic of a conventional microwave power module (MPM) based on both solid-state and microwave vacuum electronics technologies.

each of the above systems. However, due to the push for developing small satellites with enhanced system capabilities/ performance at lower cost, it is advantageous to develop a single wideband, reconfigurable high-power, high-efficiency SSMPM that can operate at multiple frequency bands depending on the need at any given time. Innovations in compound semiconductor materials, devices, and circuits to increase the functionality and reconfigureability of RF systems are reported in [6], [7].

In this paper, we present as a proof-of-concept (POC) the design, integration, and performance of a novel reconfigurable GaN based fully solid-state MPM. The module synergistically integrates diplexers, pre-amplifiers, multistage medium power amplifiers (MPAs), SPDT switches, and CW/Pulsed high power amplifiers (HPAs) with a voltage sequencer, a DC blanking controller, and a low voltage electronic power conditioner. The POC SSMPM operates at both S-band and X-band to serve multiple roles. The SSMPM can be reconfigured to deliver $\mathrm{P}_{\text {sat }}$ of $39 \mathrm{dBm}(8 \mathrm{~W} \mathrm{CW})$ at S-band, $\mathrm{P}_{\text {sat }}$ of $46 \mathrm{dBm}$ (40 W CW) at Xband, and $\mathrm{P}_{\text {sat }}$ of $>50 \mathrm{dBm}$ (>100 W Pulsed) at X-band.

\section{Solid-StATe Microwave Power Module}

\section{Module Design and Mode of Operation}

The SSMPM layout is schematically illustrated in Fig. 2. The typical frequencies at S-band and X-band for TT\&C and telecommunications are $2.2 \mathrm{GHz}$ and $8.4 \mathrm{GHz}$, respectively. At the input, a diplexer (D) selects either the S-band or the X-band signal to be processed. The two pre-amplifier stages are GaAs based MMIC amplifiers that amplify the S-band and the X-band output signals from the diplexer respectively, to a level appropriate to drive the common multistage MPA. The switch is a non-reflective silicon single-pole, double throw (SPDT) device. The multistage MPA is high efficiency GaN HEMT PAs with output power $\left(\mathrm{P}_{\text {out }}\right)$ sufficient to drive the HPA stage. The HPAs are also high-efficiency GaN HEMT PAs that provide CW or pulsed power. 


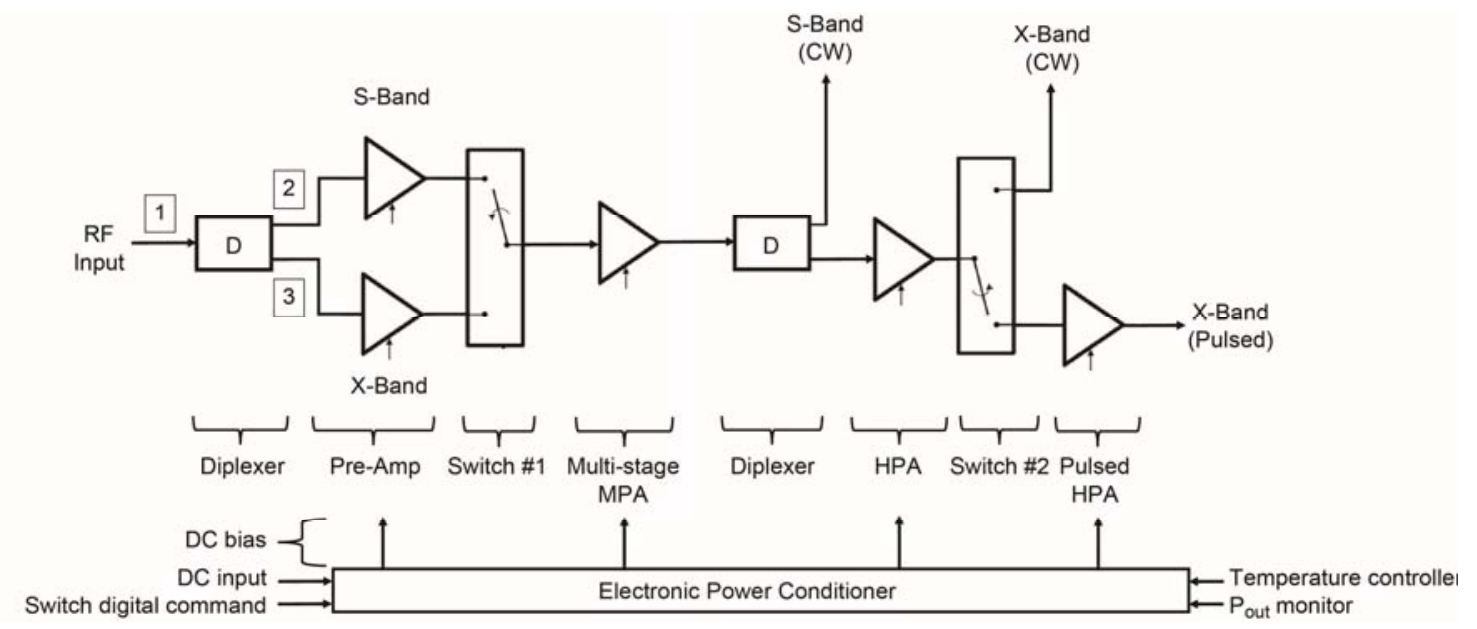

Fig. 2. Schematic of a fully solid-state microwave power module (SSMPM) based on high power GaN MMIC Power Amplifiers.

An electronic power conditioner (EPC) provides the gate and drain voltages and currents for the above amplifier stages. The EPC is a DC-to-DC power convertor that transforms the spacecraft bus voltage typically in the range of $+21 \mathrm{~V}$ and $+35 \mathrm{~V}$ into regulated voltages required by the amplifier stages. In addition, a DC power management circuit is included to manage the correct power-up and power-down sequence. That is to ensure that the negative gate voltages are applied before the positive drain voltages are applied to turn the amplifiers ON. Furthermore, a DC blanking control is also provided to quickly turn the amplifiers OFF if a fault condition arises. Moreover, a RF output monitor such as a temperature sensor or a detector/reference diode pair is located near or on the highpower GaN die, in the output stage, to monitor for an over temperature condition. The detector/reference diode pair also monitors the RF output power level. The packaged SSMPM unit is conduction cooled.

\section{MEAsuRED RESULTS}

In this section, the measured performance of the diplexer, pre-amplifiers, SPDT switch, MPA, and HPA under CW and pulsed operating conditions are presented.

\section{A. Diplexer}

The diplexer is Marki Microwave Model DPX-4. The measured insertion loss from the input to the output low-pass (LP) (S-band) and the high-pass (HP) (X-band) coaxial ports are on the order of $0.8 \mathrm{~dB}$ and $1.0 \mathrm{~dB}$ at the S-band and X-band respectively. These results are presented in Fig. 3.

\section{B. Pre-Amplifiers}

The two Avantek pre-amplifiers, model AWT-6035 operating from 2-6 GHz and model AMT-12436 operating from 7-12.4 GHz, each provides a gain of about $50 \mathrm{~dB}$ with output power and gain presented as a function of frequency in Fig. 4.

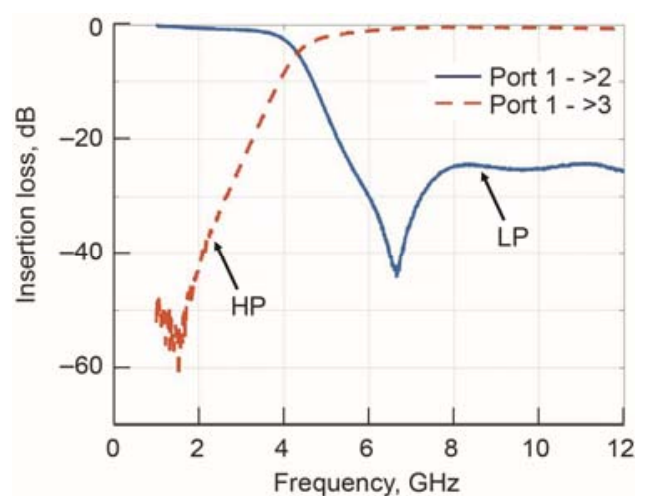

Fig. 3. Measured diplexer insertion loss at the low-pass (S-band) and high-pass (X-band) coaxial ports.

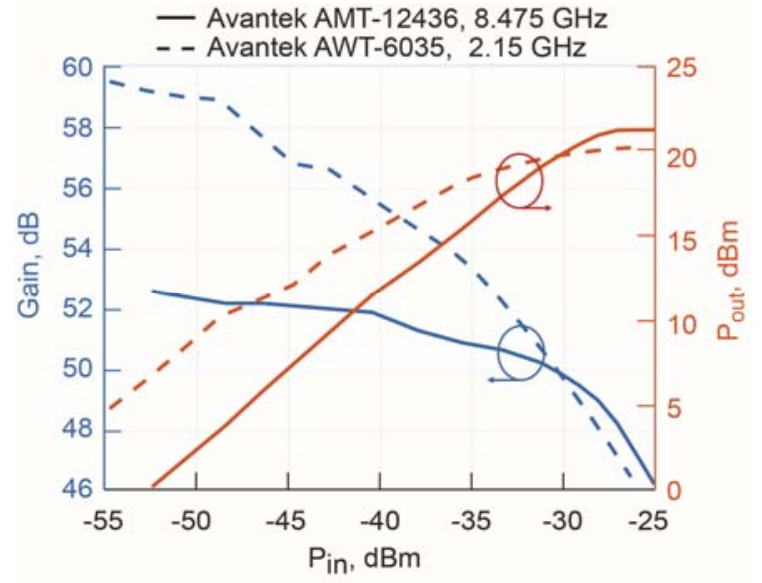

Fig. 4. Measured output power and gain as a function of the input drive at S-band (2.15 GHz) for Avantek AWT-6035 with $\mathrm{V}_{\mathrm{D}}=12 \mathrm{~V}, \mathrm{I}_{\mathrm{DQ}}=310 \mathrm{~mA}$ and at X-Band $(8.475 \mathrm{GHz})$ for Avantek AMT-12436 with $\mathrm{V}_{\mathrm{D}}=12 \mathrm{~V}, \mathrm{I}_{\mathrm{DQ}}=350 \mathrm{~mA}$. 


\section{SPDT Switch}

The switch is an Analog Devices Model HMC1118 that operates over a wideband (9 kHz-13 GHz), which allows reconfiguring the SSMPM to function at either S- or X-band. The measured insertion loss of the switch when the two inputs are sequentially turned $\mathrm{ON}$ is on the order of 0.6 and $1.4 \mathrm{~dB}$ at S-band and X-band, respectively.

The measured isolation between the input and the two output ports of the switch is greater than $50 \mathrm{~dB}$ and $40 \mathrm{~dB}$ at S-band and X-band, respectively.

\section{Wideband GaN Multistage Medium Power Amplifier (MPA)}

The first stage of the wideband MPA is built with a Qorvo Model TGA2214-CP GaN MMIC that operates from 2-18 GHz. The second stage of the MPA is built with Analog Devices Model HMC1087F10 GaN MMIC that operates from 2-20 $\mathrm{GHz}$. The measured $\mathrm{P}_{\text {out }}$ and gain of each stage at S-band (2.15 $\mathrm{GHz})$ and $\mathrm{X}$-band $(8.475 \mathrm{GHz})$ as a function of input power are presented in Fig. 5(a). The corresponding measured PAEs are presented in Fig. 5(b). The $\mathrm{P}_{\text {sat }}$ of the first stage is $37 \mathrm{dBm}$, the corresponding gain is $20 \mathrm{~dB}$, and the PAE is $25 \%$. The $\mathrm{P}_{\text {sat }}$ of the second stage is $39.5 \mathrm{dBm}$, the corresponding gain is $8.8 \mathrm{~dB}$, and the PAE is $27 \%$.

\section{E. GaN CW High-Power Amplifier (HPA)}

The CW HPA stage is configured either as a single Sumitomo Model SGK77850-30A GaN MMIC amplifier or as two SGK77850-30A amplifiers in a balanced configuration. The balanced amplifier, with topology shown in Fig. 6 employs hybrid couplers at the input and output to divide and combine the power, respectively. The measured results are presented in Fig. 7. The $\mathrm{P}_{\text {sat }}$ of the single HPA is $43.5 \mathrm{dBm}$, the corresponding gain is $7.9 \mathrm{~dB}$, and the PAE is $36.7 \%$. The $\mathrm{P}_{\text {sat }}$ of the balanced HPA is $46.7 \mathrm{dBm}$, the corresponding gain is $6.8 \mathrm{~dB}$, and the $\mathrm{PAE}$ is $34 \%$.

\section{F. GaN Pulsed High-Power Amplifier (HPA)}

The pulsed HPA is realized with a Cree Model CGHV96100F2 GaN MMIC. The measured characteristics under pulsed operating conditions is presented in Fig. 8. The $\mathrm{P}_{\text {sat }}$ of the pulsed HPA is $50.86 \mathrm{dBm}$, the corresponding gain is $9.86 \mathrm{~dB}$, and the PAE is $34.5 \%$.

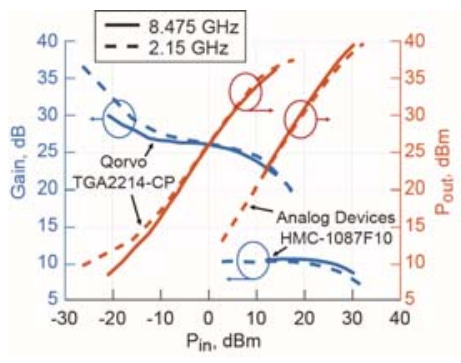

(a)

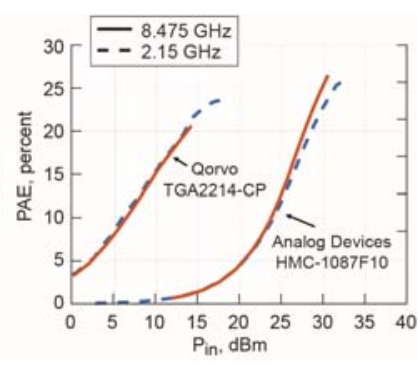

(b)
Fig. 5 (a) Measured output power and gain as a function of the input drive, and (b) Measured PAE as a function of the input power, for the MPA $1^{\text {st }}$ stage with TGA2214-CP: $\mathrm{V}_{\mathrm{D}}=22 \mathrm{~V}, \mathrm{~V}_{\mathrm{G}}=-2.3 \mathrm{~V}, \mathrm{I}_{\mathrm{DQ}}=600 \mathrm{~mA}$ and $2^{\text {nd }}$ stage with HMC1087F10: $\mathrm{V}_{\mathrm{D}}=28 \mathrm{~V}, \mathrm{~V}_{\mathrm{G}}=-2.3 \mathrm{~V}, \mathrm{I}_{\mathrm{DQ}}=850 \mathrm{~mA}$.

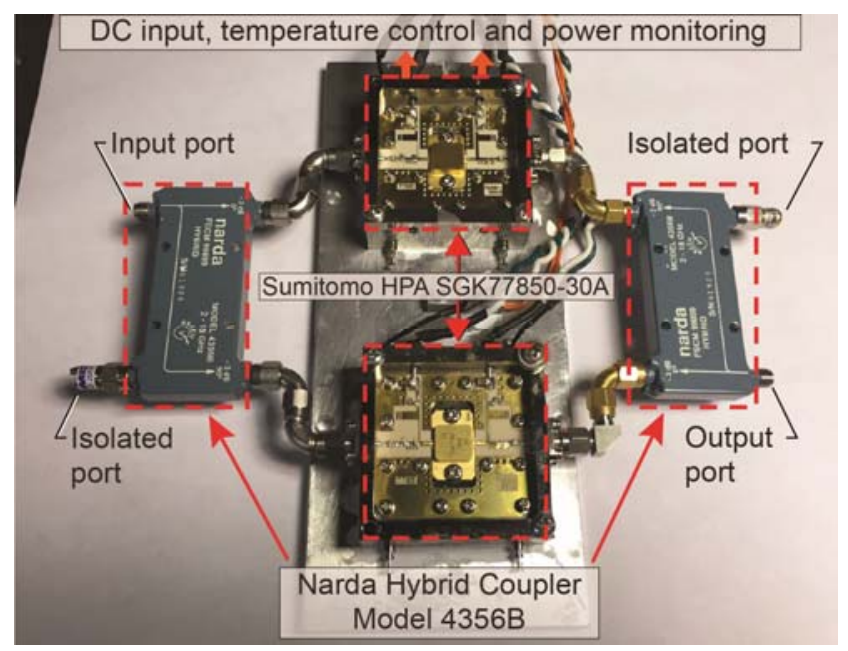

Fig. 6. Balanced amplifier configuration consisting of two Sumitomo SGK77850-30A HPA and Narda Model 4356B hybrid couplers.

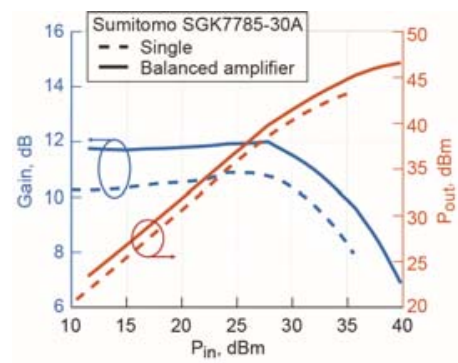

(a)

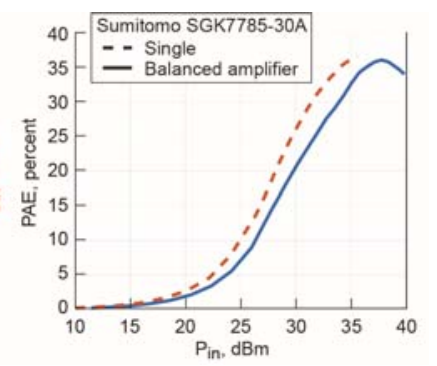

(b)
Fig. 7 (a) Measured output power as a function of the input drive, and (b) Measured PAE as a function of the input drive at X-band $(8.475 \mathrm{GHz})$, for a single Sumitomo SGK77850-30A with $\mathrm{V}_{\mathrm{D}}=24 \mathrm{~V}, \mathrm{~V}_{\mathrm{G}}=-2.2 \mathrm{~V}, \mathrm{I}_{\mathrm{DQ}}=1750 \mathrm{~mA}$ and balanced amplifier with $\mathrm{V}_{\mathrm{D}}=24 \mathrm{~V}, \mathrm{~V}_{\mathrm{G}}=-2.2 \mathrm{~V}, \mathrm{I}_{\mathrm{DQ}}=3500 \mathrm{~mA}$.

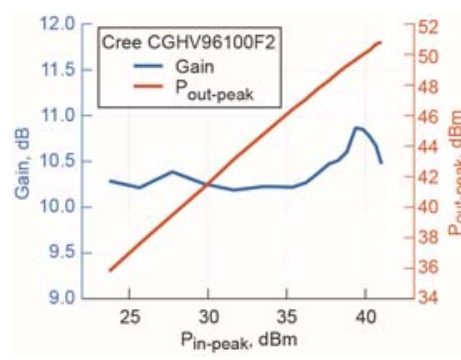

(a)

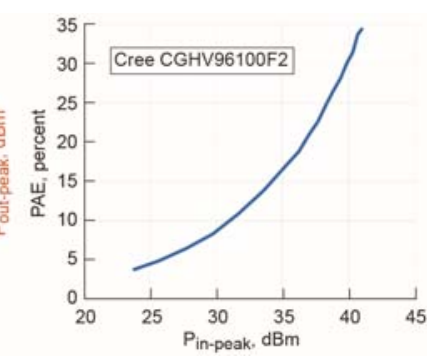

(b)
Fig. 8 (a) Measured peak output power and gain as a function of the input drive, and (b) Measured peak PAE as a function of the input drive at X-band $(8.475 \mathrm{GHz})$, for Cree CGHV96100F2 under pulsed conditions, duty cycle = $10 \%$, pulse width $=100 \mu$ sec, $\mathrm{V}_{\mathrm{D}}=40 \mathrm{~V}, \mathrm{~V}_{\mathrm{G}}=-2.2 \mathrm{~V}, \mathrm{I}_{\mathrm{DQ}}=1000 \mathrm{~mA}$.

\section{PROOF-OF-CONCEPT (POC) RECONFIGURABLE SSMPM}

A POC bread board version of the fully assembled reconfigurable SSMPM with the diplexer, pre-amplifers, SPDT switch, MPA, and HPA presented earlier is shown in Fig. 9. The measured output power of the end-to-end S-band, X-band (CW), and X-band (Pulsed) chains are presented in Fig. 10. The signal pathways are shown in the inset in Fig. 10. The results indicate that the S-band CW chain of the SSMPM delivers $\mathrm{P}_{\text {sat }}$ 


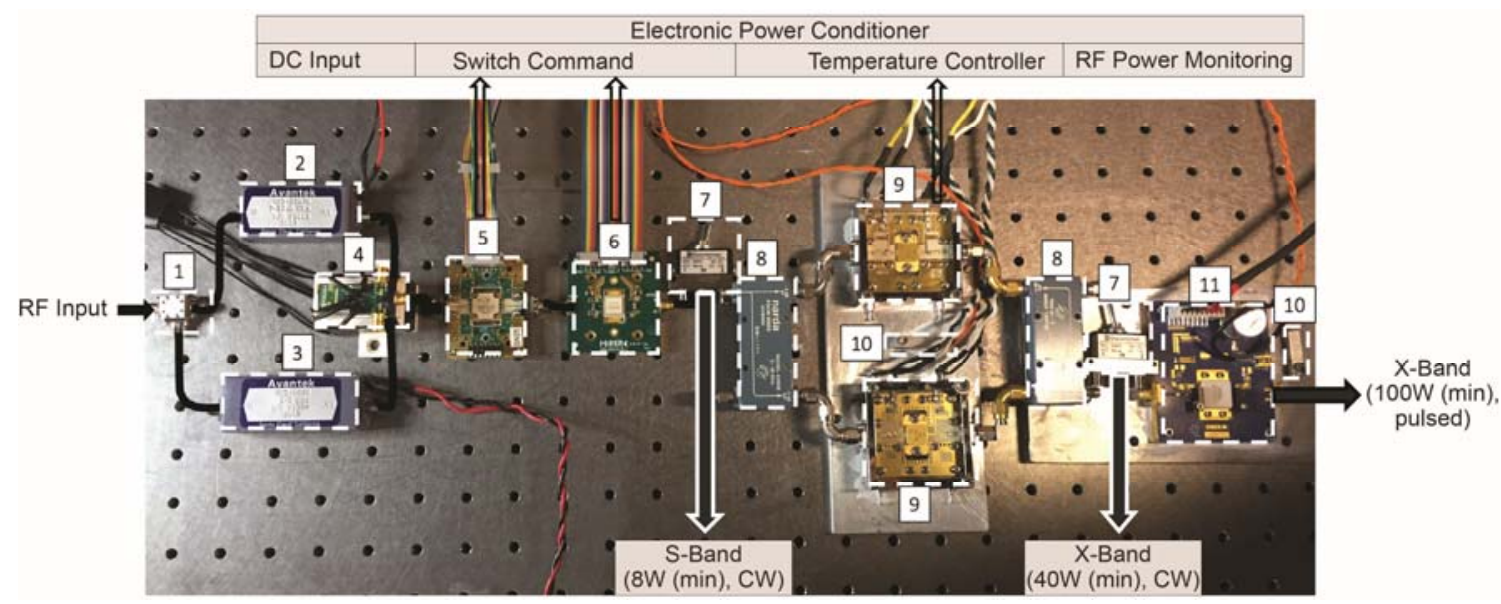

Fig. 9. A POC bread board version of the fully assembled reconfigurable SSMPM. (1) Marki Microwaves DPX-4, (2) Avantek AMT-12436, (3) Avantek AWT6035, (4) Analog Devices HMC1118 SPDT, (5) Qorvo TGA2214-CP, (6) Analog Devices HMC1087F10, (7) Ducommun 2SM001, (8) Narda 4356B Hybrid Coupler, (9) Sumitomo SGK7785-30A, (10) Thermocouple, (11) Cree CGHV96100F2.

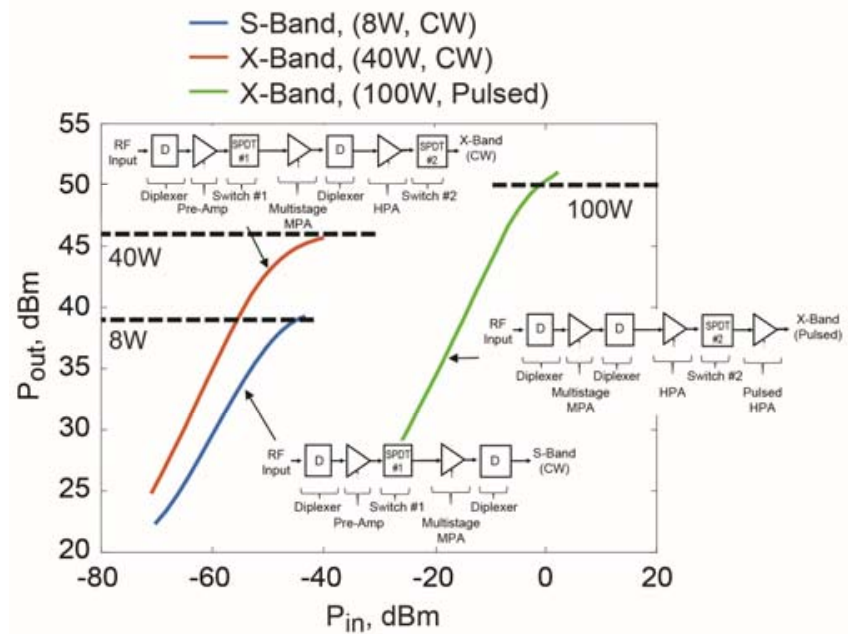

Fig. 10 Measured output power as a function of the input drive for the S-band (CW), X-band (CW), and X-band (Pulsed) signal pathways that are shown in the inset.

of $39 \mathrm{dBm}(8 \mathrm{~W})$ for TT\&C, the X-band CW chain delivers $\mathrm{P}_{\text {sat }}$ of $46 \mathrm{dBm}(40 \mathrm{~W})$ for telecommunications, and the X-band pulsed chain without the pre-amplifiers delivers $\mathrm{P}_{\text {sat }}$ of $>50$ $\mathrm{dBm}(>100 \mathrm{~W})$ for radar applications.

\section{CONCLUSION AND DISCUSSIONS}

The paper presents the design, integration, and performance of a novel reconfigurable, GaN MMIC based, fully solid-state microwave power module (SSMPM). The characterization of the individual components as well as the end-to-end performance of each of the S-band and X-band chains of the SSMPM are presented. These results indicate that the S-band CW chain can deliver $\mathrm{P}_{\text {sat }}$ of $39 \mathrm{dBm}(8 \mathrm{~W})$ for TT\&C, the $\mathrm{X}$-band CW chain can deliver $\mathrm{P}_{\text {sat }}$ of $46 \mathrm{dBm}(40 \mathrm{~W})$ for telecommunications, and the $\mathrm{X}$-band pulsed chain without the pre-amplifiers can deliver $\mathrm{P}_{\text {sat }}$ of $>50 \mathrm{dBm}(>100 \mathrm{~W})$ for radar applications.

Our link budget calculations indicate that the SSMPM with $\mathrm{P}_{\text {sat }}=40 \mathrm{~W}$ when coupled to a $10 \mathrm{~cm} \mathrm{X-band} \mathrm{transmit} \mathrm{antenna}$ on a low Earth orbiting $(900 \mathrm{~km})$ satellite can close a $1 \mathrm{Gbps}$ (QPSK) data downlink to a $1 \mathrm{~m}$ receive antenna on ground with $3 \mathrm{~dB}$ margin. Leveraging upon compound semiconductor devices and novel materials [7] will enable the monolithic heterogeneous integration of GaN plus CMOS for realization of a compact SSMPM. The above results indicate that a single SSMPM is capable of being dynamically reconfigured to serve multiple roles such as an amplifier for TT\&C, telecommunications, and radar onboard future Earth and planetary exploration spacecrafts.

\section{REFERENCES}

[1] J.X. Qiu, et al., "Vacuum Tube Amplifiers," IEEE Microwave Magazine, Vol. 10, No. 7, pp. 38-51, Dec 2009.

[2] R.N. Simons and E.G. Wintucky, U.S. Patent No. 9,041,469 B1, "HighEfficiency Power Module,” May 26, 2015.

[3] M.J. Rosker, et al., "DARPA's GaN Technology Thrust," 2010 IEEE MTT-S International Microwave Symposium Digest, pp. 1214-1217, Anaheim, CA, May 23-28, 2010.

[4] R. Chu, et al., "V-Gate GaN HEMTs for X-band Power Applications," IEEE Electron Device Letters, vol. 29, no. 9, pp. 974-976, Sept 2008.

[5] R.T. Kemerley, et al., "Impact of Wide Bandgap Microwave Devices on DoD Systems,”Proc. IEEE, Vol. 90, No. 6, pp. 1059-1064, June 2002.

[6] S. Hary, T. Barton, and J. Ebel, "Reconfigurable RF Components for Multifunction RF Systems," 2016 IEEE Compound Semiconductor Integrated Circuit Symposium (CSICS) Technical Digest, pp. 20-23, Austin, TX, Oct 23-26, 2016.

[7] D.S. Green, C.L. Dohrman, A.S. Kane, and T.-H. Chang, "Materials and Integration Strategies for Modern RF Integrated Circuits," 2014 IEEE Compound Semiconductor Integrated Circuit Symposium (CSICS), pp. 1-4, La Jolla, CA, Oct 19-22, 2014. 